\title{
Reflexiones en torno a la coherencia pedagógico-didáctica de la enseñanza como práctica mediada por TIC
}

\author{
Rosanna Forestello \\ Mónica Gallino \\ (Universidad Nacional de Córdoba. Argentina)
}

\section{Resumen}

En esta comunicación presentamos, desde nuestra función como docentes responsables-tutoras y pedagogas, una sistematización de los supuestos teóricos que sustentan el diseño y desarrollo de un módulo en aula virtual destinado a la formación de postgrado de docentes universitarios, cuyo centro de preocupación son la enseñanza y el aprendizaje mediados por tecnologías, un tema que nos confronta con el abordaje de un espacio de complejidad ya que permite analizar y reflexionar sobre la interrelación de la tarea educativa con sus actores, la institución, el contexto y la implementación significativa y pertinente de las tecnologías. Sabemos que la incidencia de las nuevas tecnologías en el campo de la educación nos está indicando el surgimiento de un nuevo paradigma pedagógico-tecnológico.

En el desarrollo de este trabajo enfatizamos, por un lado, la necesidad de no perder la mirada pedagógica de las propuestas educativas mediadas por las TIC y, por el otro, resaltamos algunas categorías didácticas que sostienen las decisiones del diseño de las mismas.

\section{Palabras clave \\ Enseñanza - Aprendizaje - TIC - Mediación - Prácticas Docentes.}

\section{Summary}

In this communication we present, from our roles as responsible teachers, tutors and pedagogues, a systematization of the theoretical assumptions that sustain the design and development of a module in a virtual classroom aimed at post-graduate studies for university students, whose centre of interest is technology-mediated teaching and learning; a topic that confronts us with the treatment of a space of complexity, since it allows us to reflect on the interrelation of the educational activity with its participants, the institution, the context, and the significant and relevant implementation of technologies. We know that the influence of new technologies in the field of education is indicative of the appearance of a new technological and pedagogical paradigm. 
In the development of this work we emphasize, on the one hand, the need not to lose the pedagogical view of ICT-mediated educational proposals, and on the other, we highlight some didactic categories that support the decisions of their design.

\section{Key words}

Teaching - Learning - ICT - Mediation - Teaching practices.

\section{Breve caracterización de la propuesta}

En esta comunicación presentamos, desde nuestra función como docentes responsables-tutoras y pedagogas, una sistematización de los supuestos teóricos que sustentan el diseño y desarrollo de un módulo en aula virtual destinado a la formación de postgrado de docentes universitarios. El centro de preocupación del módulo es la enseñanza y el aprendizaje mediados por tecnologías. Este módulo es parte de la propuesta del plan de estudios de la Maestría "Procesos Educativos mediados por Tecnologías" implementado en el marco del Centro de Estudios Avanzados (CEA) de la Universidad Nacional de Córdoba. Desde hace tres años venimos realizando esta propuesta educativa y sentimos que queremos, a través de este trabajo, resaltar y explicitar algunas categorías conceptuales pedagógicas y didácticas que sostienen las decisiones al incorporar tecnologías en las propuestas de enseñanza, ámbito donde se ponen de manifiesto una gama de procesos, teorías -implícitas y explícitas-, actitudes y enfoques que desvelan las fracturas o articulaciones entre los supuestos teóricos y las prácticas pedagógicas.

El propósito central de la misma es ofrecer una formación teórica y herramientas prácticas que habiliten al docente universitario para intervenir de manera innovadora, con criterios propios y nuevas metodologías, implicando un replanteo tanto de mediaciones tecnológicas como del tratamiento de contenidos propios de las distintas áreas disciplinares.

En el marco institucional planteado con anterioridad y dentro de la propuesta curricular de la Maestría se desarrolla el Módulo La enseñanza y el aprendizaje del cual somos docentes responsables y tutoras.

El propósito central del módulo es resignificar los procesos de enseñanza, de aprendizaje y de comunicación en entornos tecnológicos, a fin de lograr una nueva configuración didáctica a la luz de nuevos aportes científicos, pedagógicos y de tecnologías aplicadas a la educación.

Los contenidos que orientan el trabajo al interior del módulo hacen referencia a poder analizar, resignificar los procesos de enseñanza, de aprendizaje y comunicación construidos desde las configuraciones didácticas de nuestras 
clases, en las cuales la preocupación central sigue siendo democratizar el acceso al conocimiento a partir de buenas prácticas de enseñanza.

El módulo, desde el comienzo, fue definido como un curso teórico-práctico. Se parte del supuesto que es difícil apropiarse de este tipo de contenidos si al mismo tiempo no se realiza una práctica de los aprendizajes en un contexto virtual.

Por ello la propuesta metodológica planteada permitió a los alumnos acercarse conceptualmente a contenidos relacionados con la enseñanza y el aprendizaje mediados por tecnologías desde un enfoque socio-cultural como también a formas de trabajo que impactan en la práctica educativa de los que participan en ella.

La modalidad de trabajo virtual propuesta se basó en un enfoque sobre la enseñanza y el aprendizaje específicos de procesos educativos a distancia, en línea. En esta propuesta la enseñanza y el aprendizaje virtuales se apoyan en dos modalidades de trabajo: trabajo individual y trabajo grupal. En ellas el papel del docente virtual -el tut@r- es el de un guía, que acompaña al participante tanto a nivel individual como colectivo, en la construcción del conocimiento así como en el desarrollo de ciertas habilidades.

\section{La incidencia de las TIC en las propuestas educativas}

Todo quehacer educativo es una tarea de compromiso humano social que compromete a quien lo realiza y guarda intrínsecamente una relación intencional que obliga moralmente a sus responsables. Si sumamos a esto el hecho de que vivimos una etapa de cambios tecnológicos, sociales, culturales, políticos y económicos, se hace aún más perceptible la demanda de una exigencia de transformaciones de calidad en la educación.

Los docentes se desempeñan como educadores en un escenario atravesado por complejas demandas vinculadas a un imperativo tecnológico que se presenta como neutral y absoluto. En este contexto resulta imprescindible ofrecer a los profesores las oportunidades de desarrollar las herramientas teóri$\mathrm{co/conceptuales} \mathrm{necesarias} \mathrm{para} \mathrm{analizar} \mathrm{este} \mathrm{imperativo} \mathrm{tecnológico} \mathrm{y} \mathrm{asumir}$ lugares de liderazgo al interior de las instituciones educativas que recuperen el tema de la tecnología desde una perspectiva crítica.

Este escenario enfrenta a los educadores a pensar y pensarse en relación con nuevos desafíos epistemológicos, culturales y políticos. Consideramos que la formación docente debe ofrecer las herramientas necesarias para abordar estos desafíos y desde allí se podrán ir definiendo propuestas reflexivas para las prácticas de la enseñanza influenciadas por los desarrollos tecnológicos.

Nos interesa desentrañar la compleja trama educativa que implican los procesos de enseñanza y de aprendizaje mediados por tecnologías, lo que supone un análisis de las incidencias de este aspecto en la relación docente- 
alumno, en la comunicación educativa y en los cambios que los nuevos entornos provocan en la educación toda.

La tecnología se incorpora ya no como mero producto sino como entorno de procesos y recursos.

Desde esta perspectiva implica tomar conciencia de que existe una trama que imbrica a la educación y la tecnología desde una perspectiva en la que es importante distinguir que ni una ni la otra son neutras.

Ellas transparentan narrativas y aplicaciones que explicitan la concepción implícita que de ellas sostenemos. El diseño de un proyecto didáctico que integra tecnología implica siempre partir de ideas, teorías que concebimos interiormente -conocimiento tácito- y que luego damos forma en su aplicación práctica. ¿Qué sujeto pedagógico deseamos potenciar? ¿Qué conocimientos, competencias? ¿Qué lugar ocupa la tecnología en estas prácticas? ¿Cómo influyen en el diseño formativo?

"Si se sostiene como principio para la enseñanza enseñar a partir de lo que ya se sabe, las tecnologías ocuparán diferentes lugares según el reconocimiento que de ellas se tenga y sus potencias para facilitar la disponibilidad de la información" (Litwin, 2005, p. 23).

Este contexto impone transformaciones, nuevos procesos y resignificaciones. Refiere a un tipo de propuesta pedagógica en la que la educación ya no podrá estar dirigida a la transmisión de conocimientos sino a desarrollar la capacidad de reflexionar, producir y utilizar conocimientos de orden superior. El rol docente, entonces, se traduce en un acompañante cognitivo y el proceso de comunicación en una experiencia de interacción como intercambio y negociación de sentido, en ambientes virtuales y colaborativos de aprendizaje. En este marco, se impone resignificar roles y procesos apuntando a una nueva configuración didáctica que perfile una buena enseñanza, un buen aprendizaje, sino caeríamos en puro activismo, es decir, realizar actividades con las computadoras, pero sin continuidad ni significado educativo. Es evitar lo que J. Sancho (2006) califica como el "vacío pedagógico" de muchas propuestas, proyectos y experiencias de aplicación de las TIC a la educación.

\section{La enseñanza como acto educativo}

La enseñanza no es un proceso rígido, uniforme, sino multivariado y contextualizado, es un proceso complejo que posee "una engañosa simplicidad" y por ello no implica el "hacer las cosas a mi manera ó de cualquier manera", sino que, al ser un proceso pedagógico, ideológico, disciplinar y ético, la enseñanza se concibe en un marco democrático para facilitar a sus miembros el acceso a determinados conocimientos, competencias y valores, que se consideran importantes en su integración crítica y autónoma al seno de una sociedad compleja. Pero es conveniente remarcar que los conocimientos, competencias 
y valores que se tienen que enseñar, convivir, compartir y aprender, son los que podrían no ser adquiridos, o serlo sólo parcialmente. Hay muchas cosas que no se enseñan, pero que se aprenden.

Es por ello que hablamos de procesos de enseñanza dirigidos intencionalmente a generar, potenciar, posibilitar el aprendizaje.

Para poder comprender las particularidades de la enseñanza y de la relación pedagógica que se entabla es necesario recordar que estamos frente a un hecho educativo y son válidas las consideraciones propias de este campo. Pero a su vez, también nos encontramos con una manera diferente de producir la enseñanza, el aprendizaje y la relación pedagógica, lo cual requiere de conocimientos, procedimientos y actitudes especiales en los integrantes del sistema. Tarea que adquiere relieves particulares en tanto enseñanza con TIC.

No podemos desconocer que en la formación de los docentes han impactado modelos pedagógicos que han generado ciertas tradiciones o filosofías en las prácticas áulicas. En este sentido, todo proyecto o práctica de enseñanza realizadas con las TIC debiera planificarse en una perspectiva metodológica que asuma los planteamientos y principios de un modelo y método educativo apoyado en las teorías que han inspirado al conocimiento pedagógico construido a lo largo del siglo XX.

Tres son los grandes modelos pedagógicos (1) que podemos distinguir:

- Un primer modelo, fundado en las pedagogías tradicionales, cuyo eje se centra en el conocimiento construido consolidado y acabado. Se arraiga en las tradiciones normalizadora-disciplinadora, la académica y la eficientista, ya que las mismas responden a matrices compartidas y se pueden señalar rasgos comunes a las mismas. En principio, se destaca un discurso prescriptivo acerca del deber ser del docente, entendiéndose su función más como funcionario del estado que como educador o profesional. Tiene una noción de neutralidad de la escuela y del conocimiento, heredada de la modernidad y del positivismo y la visión homogénea y homogeneizadora de la realidad. Según este modelo, el docente cumple un rol activo, de transmisión lineal y homogénea y el alumno asume un rol pasivo, de escucha y ejercitación.

- Un segundo modelo, basado en las teorías en las que el eje se centra en el educando y sus intereses; la estructura propia del saber pasa a un segundo plano y las acciones del educador se limitan a disponer las condiciones para que el alumno organice sus estructuras o aprenda a investigar la realidad. Se incluyen las tendencias hermenéutico-participativas y las huellas que ha dejado la escuela nueva, de allí que las denominaremos teorías emergentes. Se propone la modificación de la organización institucional, los rituales, las formas y condiciones de trabajo.

- Un tercer modelo, apoyado en las teorías críticas, el cual se centra en la recuperación de contenidos significativos en la enseñanza desde un en- 
foque de crítica social e histórica, entendiendo la transmisión de la cultura como necesaria para la transformación social parte de un enfoque de crítica social e histórica. Se considera la no-neutralidad de los contenidos, las prácticas sociales y la enseñanza. Se destaca la importancia de la función docente en la transmisión del conocimiento como herramienta idónea de intervención y transformación social a través de un vínculo pedagógico no autoritario.

En estas tres posturas aparecería implícita alguna forma de "transmisión de cultura" pero cada teoría le asigna un valor diferente dentro de su marco conceptual.

Desde el modelo tradicional, enseñar y transmitir pueden considerarse sinónimos ya que la transmisión, en esta concepción, implicó trasladar contenidos consolidados y sistematizados y lograr que el alumno los incorporara. En el ámbito de la educación a distancia ha ocurrido que más de una institución que surgió como propuesta de innovación y cambio, finalmente termine siguiendo un modelo en esta línea. En muchos casos, las aulas virtuales, las propuestas en línea y los materiales didácticos conservan la magistralidad típica de este modelo, es decir, aquélla que ve al profesor como el poseedor del conocimiento, y por tanto, agregando tecnología, mantienen el carácter tradicional de "cátedra".

En lo que concierne a la interacción, podemos decir que la técnica de exposición magistral no lleva aparejada la comunicación bidireccional. Esto significa que el modelo descansa en la presentación eficaz, convincente y magistral de los contenidos que realiza el profesor, estando considerada la intervención del estudiante para aclarar dudas relativas a los contenidos presentados por el docente.

Se puede entender esta permanencia del modelo, por este arraigo cultural que no permite a los docentes, aún cuando posean conocimientos teóricos de otros modelos, desprenderse de la tendencia academicista.

Desde el modelo centrado en las teorías emergentes, el enseñar está ligado a la construcción de las estructuras mentales, mientras que la transmisión del conocimiento pasaría a un segundo plano, adquiriendo una función instrumental, funcional y de mediación entre el docente y el alumno.

El paradigma crítico se acerca, en la valoración de la transmisión del conocimiento, al paradigma tradicional, entendiéndolo como necesario para la transformación social, pero desde una perspectiva de crítica social y política.

Esto implica concebir a la educación como un proceso que supera la simple transmisión de información para transformarla en una dinámica activa y de autogestión tanto de la enseñanza como del mismo aprendizaje. Por lo cual, es una tarea que se vive y se crea "desde adentro", es decir desde los procesos de interacción y comunicación que se dan en el "aula", y a la vez está determinada "desde afuera", en cuanto que forma parte de la estructura y finalidades de una institución y, más aún, de los requerimientos de una sociedad. 


\section{4. ¿Por qué pensar en el diseño y desarrollo de propuestas educativas mediadas con TIC?}

Podemos pensar algunas razones que resultan significativas, posibles respuestas vinculadas con aspectos centrales de la tarea de enseñar.

Porque como docentes necesitamos ingresar en el desafío que las nuevas tecnologías generan hoy en los procesos de construcción del conocimiento y de circulación de la información. La explosión de Internet, a comienzos de la década del 90, puso de manifiesto la enorme transformación que las nuevas tecnologías generan en los modos de acceder a la información y trabajar con ella en la construcción del conocimiento. Pero no sólo las fuentes de información se ven transformadas. También las herramientas que utilizamos para interactuar con ellas son nuevas y nos permiten hoy acceder y construir textos electrónicos, documentos de imágenes y sonidos, generando productos de nuevo tipo. También las TIC están transformando el escenario y los modos en los que las comunidades académicas y científicas trabajan. Es por ello que los procesos de construcción, circulación y legitimación del conocimiento se ven entretejidos con los procesos de construcción, circulación y legitimación de las nuevas tecnologías en diversos ámbitos sociales y académicos. Por supuesto, con las transformaciones vienen los desafíos y las oportunidades. Aquí nos preguntamos: ¿qué aprendemos cuando utilizamos estas nuevas fuentes de información y qué necesitamos aprender?, ¿qué significa conocer en la era de la información?, ¿de qué manera construimos criterios para validar la información?, ¿cómo podemos potenciar las nuevas herramientas de comunicación para acercar a nuestros alumnos a las comunidades académicas y científicas?, ¿cuál es el impacto que producen en las aulas el descentramiento, la deslocalización y la diseminación de la información? No podemos negar que se abren numerosos interrogantes a partir de las TIC y su relación con los procesos del conocer, es precisamente la existencia de la relación TIC-conocimiento, lo que nos invita a ocuparnos de estos interrogantes como docentes universitarios.

Porque como docentes necesitamos comprender los múltiples modos en los que nuestros alumnos se comunican, se expresan y se acercan a conocer el mundo. Hoy existe en el mundo de la cultura una nueva ecología comunicativa. Los teléfonos celulares, la computadora, los videojuegos, la televisión por cable, los reproductores de música e imágenes, Internet, las redes sociales, las cámaras fotográficas digitales, por sólo nombrar algunos, son parte de los modos en los que podemos comunicarnos e interactuar. Reconociendo diferencias socioculturales y económicas que impactan en el acceso y uso, los niños y jóvenes de hoy viven en un mundo comunicacional sustantiva y cualitativamente diferente al que nosotros conocimos en nuestra adolescencia.

En los 90 Internet era, básicamente, una gran y enorme biblioteca. Ya no vemos a la Web de esa manera. Hoy es algo diferente. Es una especie de espacio de trabajo colaborativo, de construcción de conocimiento, de gente pro- 
duciendo y publicando, participando en redes sociales con diferentes objetivos. Es también un espacio de creatividad, de comunicación, de expresión personal, de exploración de identidad y subjetividad, de creación de comunidades.

Reconocemos que los tiempos y los espacios para conversar, para leer, para escribir, para pensar, se ven fuertemente atravesados por estas experiencias en esta nueva ecología comunicativa. El tiempo libre y el entretenimiento en el mundo de los nativos y de los inmigrantes digitales también se encuentran transformados por las nuevas tecnologías. ¿Cómo entran estas experiencias en las escuelas y en las aulas? ¿Cómo interpretamos los procesos de construcción de conocimiento de nuestros alumnos en relación con sus experiencias, actividades fuera de la escuela? ¿Qué conocimientos propios de las nuevas generaciones ingresan, cruzan, complementan los contenidos que se enseñan en las aulas?

Porque como docentes podemos apropiarnos de estas tecnologías como herramientas, recursos para abrir puertas que nos permitan seguir enseñando. Los cambios generan incertidumbres, oportunidades y desafíos para seguir diseñando, implementado diferentes propuestas de enseñanza. Podemos pensar en las TIC, apropiándonos de ellas como herramienta para pensar y experimentar nuevos entornos de trabajo, nuevas actividades de aprendizaje, nuevas propuestas de enseñanza. En síntesis, como docentes es posible vincularnos con las nuevas tecnologías de manera que las mismas nos permitan reflexionar acerca de lo que hacemos, cómo lo hacemos y cómo podemos hacerlo. Es necesario seguir planteándonos a la hora de diseñar una propuesta educativa: ¿cuál será el proyecto final de este curso?, ¿cómo quiero que trabajen los alumnos colaborativamente?, ¿cuáles son las actividades que quiero para mis alumnos?, ¿qué tipo de intercambios comunicativos me interesa promover?, ¿cómo voy a generar un involucramiento por parte de ellos? Podemos formularnos preguntas que nos permiten mirar a esas clases de manera diferente. Podemos apropiarnos de la tecnología para crear proyectos que se acerquen a la educación que deseamos construir en nuestras aulas. Pero recordemos lo que nos dice Manuel Área Moreira (2001, p. 389), que "los ordenadores no cambian la educación, pero los profesores sí", que nos recuerda que no es "la tecnología" la que nos permite producir estos cambios, sino nuestra decisión de pensar, de imaginar con y a través de ella. Nuestro compromiso de producir propuestas con un fuerte sentido educativo. Esta nueva relación epistemológica y comunicativa debe ser nuestro norte de trabajo como docentes.

\section{Pensando la enseñanza desde algunas categorías didácticas}

Es el momento para adentrarnos en la realidad del proceso de enseñanza mediado por tecnologías. Sin embargo es necesario aclarar que los principios que regulan y participan en este proceso se fundan en las preocupaciones centrales de la Didáctica, más allá de la implementación o no en entornos 
tecnológicos. Éste es el punto de partida para hacer posible que los nuevos conocimientos y las TIC puedan integrarse a las prácticas cotidianas y hacerse componentes habituales de los diseños de planificación áulica, lo que significa preocuparnos por la enseñanza y las prácticas docentes, las estrategias de intervención que elaboran los docentes, las interacciones de los sujetos con el conocimiento mediado por las TIC, el contenido a través de las TIC, la relación entre construcción metodológica y el uso de las TIC, las actividades de enseñanza y las actividades de aprendizaje.

$Y$ entonces, no podemos dejar de formularnos preguntas como punto de partida. Siguiendo a Philip Jackson (2002, p. 17) nos preguntamos: "¿qué deben saber los docentes sobre la enseñanza?, ¿qué conocimiento es esencial para su trabajo?, ¿hay mucho que aprender o sólo un poco?, ¿es fácil o difícil?, ¿cómo se genera y confirma ese conocimiento?, ¿qué necesitan saber los docentes para poder enseñar?", a las cuales nos atrevemos agregar: ¿por qué dedicar tiempo y entusiasmo en la construcción de proyectos pedagógicos a través del uso de nuevas herramientas y recursos tecnológicos?, ¿qué significado adquieren las nuevas tecnologías de la información y la comunicación para nosotros, educadores preocupados por la buena enseñanza?

Desde el sentido común, el nexo entre enseñanza y aprendizaje es tan íntimo que la comprensión de un proceso parecería suponer la del otro. Pensar en este binomio es una categoría fundante de nuestras prácticas porque cuando uno diseña y desarrolla una situación de enseñanza lo hace pensando en la construcción de conocimiento dentro de un campo disciplinar en términos de posibilitar aprendizajes.

Pensar la enseñanza y el aprendizaje como procesos diferentes no niega que la preocupación central de la enseñanza es el aprendizaje. Nadie discutiría que es el principal y único propósito que cualquier docente tiene.

Juan Amos Comenio escribió, entre 1628 y 1632, la Didáctica Magna. En ella define magistralmente el sentido esencial de la enseñanza: "Enseñar de un modo cierto, de tal manera que no pueda menos de obtenerse un resultado. Enseñar rápidamente, sin molestia ni tedio alguno para el que enseña y para el que aprende, antes por el contrario, con el mayor atractivo y agrado para ambos".

Hay diversas concepciones de enseñanza, dependientes de concepciones de aprendizaje unas, de tradiciones históricas otras y, sobretodo, de posiciones ideológicas de lo que deba ser el papel de la educación en la sociedad. Podemos conceptualizarla desde la racionalidad técnica o como una práctica política, desde una tradición mimética o desde la tradición transformadora siguiendo la mirada de Philip Jackson (2002). Podemos posicionarnos y focalizarla como lo hace Seymour Sarason (2002) como arte de representación y abrir una nueva puerta para poder analizarla como una relación dialógica con la ayuda de Nicholas Burbules (1999). 
Aún admitiendo la diversidad de opciones respecto de las formas de entender la enseñanza es ya recurrente concebirla como actividad intencional. Sostenida sobre procesos interactivos múltiples, las prácticas de la enseñanza cobran una particular e irrepetible forma a partir de las definiciones y decisiones que el docente concreta en torno a una dimensión central y constitutiva de su trabajo: el problema del conocimiento, cómo se comparte y construye el conocimiento en el aula.

Las situaciones de enseñanza son prácticas mediadas, ponen en acto un conjunto de supuestos, definiciones, estrategias metodológicas y herramientas que promueven la relación entre docentes, alumnos y conocimiento.

La clase es un verdadero espacio educativo, es un entorno donde se establece una compleja trama de intercambios entre el conocimiento académico, el conocimiento cotidiano y el conocimiento científico, como así también entre los sucesos y "resonancias" que provoca el docente con su intervención y la participación de los alumnos, mediados tecnológicamente.

Este tipo de intercambios es lo que denominamos comunicación o diálogo didáctico, caso particular de la comunicación humana, caracterizada por involucrar tres procesos: el de enseñanza, el de aprendizaje y el de la comunicación, un objeto de conocimiento; la disciplina, un contexto situacional y tecnológico y una intencionalidad: provocar el aprendizaje.

Es evidente que esto nos remite al rol del docente como sujeto central de la tarea de enseñanza, como decisor fundamental en la construcción metodológica de la clase y en la configuración del ambiente de aprendizaje.

Es decir, constituye una alternativa superadora de la enseñanza como mero traspaso de información y de la concepción de participación simplemente como aplicación de algunas dinámicas grupales. ¿Y qué quiere decir, por lo tanto, recobrar el sentido en educación? Desde esta mirada, educar es buscar procesos con significados que involucren a docentes y alumnos, es dar sentido a lo que hacemos y a las actividades que se realizan, relacionar y contextualizar las experiencias, los contenidos. Si todo esto está ausente, la lectura, las actividades, el análisis de casos, las estrategias potenciadoras de procesos cognitivos sustanciosos, no representan nada para quien los realiza y afecta directamente el aprendizaje.

El sentido se construye en un contexto de comunicación y participación, de interlocución entre los actores del proceso.

Sin embargo, como ya expresamos, no alcanza solamente con la intencionalidad educativa sino que se requiere de un diseño, la elaboración de estrategias que partan del conocimiento de la realidad que se desea transformar y ello supone la toma de decisiones fundadas en la comprensión del perfil psicosocial de los alumnos, de los procesos que intervienen en la construcción de los conocimientos, quién, qué y cómo se seleccionan los contenidos, bajo qué presupuestos y argumentos, cómo vincular coherentemente la lógica del objeto de conocimiento con la lógica psicológica del sujeto que aprende, cuál 
es y cómo posibilitar la trama de intercambios necesarios para la construcción social del conocimiento, bajo qué formatos y a través de qué soportes viabilizar el aprendizaje, qué espacio ocupa la tecnología y para qué.

Según Alfredo Furlán (1989), "intervienen en esta propuesta cuatro componentes básicos a partir de los cuales se estructura la enseñanza: el contenido; las actividades, los recursos y las formas de interacción". La manera en cómo se relacionen estos cuatro componentes se traduce en una propuesta metodológica.

Por su parte, G. Edelstein (1997, p. 85) plantea que "la construcción metodológica deviene fruto de un acto singularmente creativo de articulación entre la lógica de los contenidos, las posibilidades de apropiación de éstos por parte de los sujetos, y las situaciones y los contextos particulares que constituyen los ámbitos donde ambas lógicas se entrecruzan".

El modo en que se piensa, organiza y realiza la intervención didácticadisciplinar gira en torno a la toma de decisiones de cómo se concibe la educación, la formación de los alumnos de acuerdo con un perfil profesional, los requerimientos sociales, y fundamentalmente con aquellas decisiones que promueven acciones y comportamientos de principio, entre otras.

En este sentido una alumna reflexiona: "Furlan y E. Remedi llevan a cabo un desarrollo de lo que implica esta estructura metodológica. Muestran que en primer lugar el docente debe construir lo que denominan 'Estructura Conceptual'. Esto es, la selección de conocimiento, es decir, de conceptos, ideas básicas, principio y teorías. Pero para lograr enseñar el contenido seleccionado, se deben buscar las formas idóneas que impliquen concebir al alumno como sujeto activo y no como mero receptor. Para lograr esto el docente debe pasar a la elaboración de la estructura metodológica de base, en ella se reorganiza la estructura conceptual para ser enseñada en función de un determinado tipo de alumno. Aquí se hace referencia a las operaciones que el docente realiza para organizar los factores y actividades que intervienen en los procesos de enseñanza y aprendizaje. Es imprescindible que los docentes seleccionen y organicen los materiales y medios a través de los cuales es presentado el contenido. Estos materiales deben permitirle al alumno operar con el conocimiento y llegar a comprenderlo mejor para poder utilizarlo en nuevas situaciones. Sin embargo, el docente debe tener la precaución de no caer en el error de organizar los contenidos en función a un determinado medio, por el contrario la construcción metodológica se realiza en base a los contenidos y desde ellos se seleccionan los medios y materiales más óptimos para el desarrollo de ese conocimiento" (Blanca, Lic. en Computación).

El docente interactúa como mediador entre el alumno y el objeto de conocimiento junto con las competencias y habilidades de formación.

Es decir, consiste en promover y acompañar el aprendizaje. Aborda la educación como un fenómeno comunicacional que exige la participación activa del alumno, centro del proceso. En la mediación se concilian "dos pasiones... 
la pasión por la ciencia, por el conocimiento y la pasión por la pedagogía" (2) integrándolas en una propuesta de discurso pedagógico que permiten procesos ricos de aprendizaje.

Es decir, que propone la conciliación entre las diferentes áreas y objetos de conocimiento y sus prácticas, por un lado, y el alumno en situación de aprendizaje, por el otro. Por ello este concepto nos remite a la figura de "puente entre...", "nexo entre..." las disciplinas y el alumno dentro del proceso educativo. Como así también entre lo que sabe y lo que no sabe, entre lo vivido y por vivir, entre la experiencia y el futuro, pero siempre significa promover y acompañar el aprendizaje del alumno con todos los productos de la imaginación y la creatividad humana.

En este sentido una alumna reflexiona: "necesitamos hoy que cada docente esté capacitado pedagógicamente y tecnológicamente, dotado de una fuerte actitud proactiva y crítica, diseñador experto de su proyecto educativo y ejecutor reflexivo y no lineal del mismo, docentes a los cuales es necesario brindarles el apoyo y acompañamiento institucional, profesional y de pares necesario para afrontar el desafío y disfrutar de los logros cognitivos en sus alumnos" (Beatriz, Ing. Electrónica).

Conocer y poder transferir a las prácticas de enseñanza este enfoque significa pensar al profesor y su propuesta educativa de enseñanza apoyada en TIC con posibilidades de:

- Preocuparse por reconocer en el sujeto que aprende, sus capacidades, potencialidades y dificultades particulares, tomando contacto con la variedad de contextos de origen.

- Posibilidad de reconstruir y dar significado a la multitud de información que obtiene en los múltiples medios de comunicación de la sociedad del siglo XXI y desarrollar las competencias para utilizar de forma inteligente, crítica y ética la información.

- Considerar que vamos en camino de cuestionar el monopolio del libro de texto como fuente única del conocimiento, lo que significa estimular la búsqueda de nuevas informaciones a través de variadas fuentes y tecnologías, así como la reflexión y el contraste crítico permanente de los datos.

- Organizar tareas y actividades que impliquen la utilización de la tecnología que demanden el desarrollo de procesos de aprendizaje colaborativo entre los alumnos de la clase.

- Visualizar la relación docente-alumno como un vínculo "asimétrico" que no implica jerarquías, sino la posibilidad de que el aprendiz pueda aprovechar los conocimientos, experiencias del experto conformados en "andamios" para el aprendizaje. Esta situación de asimetría descansa en "el acceso diverso a los resortes que permiten regular las actividades, definir los objetivos de la tarea, los pasos a seguir en su resolución, los criterios para evaluar la calidad, logros, etc. ... Los andamios provistos cumplirían fun- 
ciones como: mantener la orientación hacia las metas buscadas, subrayar aspectos críticos de la tarea, etc." (3).

- Generar estrategias de acompañamiento del aprendizaje, que estimule y que promueva reajustes durante la marcha.

- Seguimiento que no implica control sino la búsqueda de espacios de comunicación e interacción.

- Resignificar la concepción de evaluación, superando la idea tradicional que sólo "prueba" lo adquirido o logrado. Por el contrario se apunta a descubrir posibilidades de desarrollo de las capacidades superiores. En lugar de centrarse en lo que ya aprendió, atender a lo que podría lograr con ayuda del docente o sus pares.

Es concebir coherencia e integración entre la propuesta de las herramientas, recursos, entornos elegidos, en las estrategias de enseñanza, en las actividades de aprendizaje y los soportes comunicacionales poniendo el acento en las intervenciones didácticas, potenciando espacios para la construcción y co-construcción de conocimientos a través de estrategias que generen nuevas orientaciones, nuevas competencias y fundamentos para la elaboración de criterios en la gestión de los aprendizajes y la regulación del propio ritmo de avance.

Un alumno del módulo entonces reflexiona: "hoy sabemos que en general, a mayor complejidad del conocimiento sobre el que versa el aprendizaje, mayor complejidad requerirán las representaciones utilizadas para comprenderlo. (Por supuesto que esta relación no es necesariamente lineal, y en ocasiones ideas complejas pueden ser representadas de una manera sencilla). Las representaciones son, de por sí, tecnologías educativas. Incluyo en mi concepción tanto las consideradas "antiguas" como las llamadas "nuevas", recordando que pienso que estas denominaciones son puramente relativas y no obedecen a propiedades de esas tecnologías, sino que están más relacionadas con los criterios y experiencias (o inexperiencias) de quienes así las rotulan. Lo que sí interesa, es saber en qué medida las tecnologías de cualquier clase pueden ser útiles para estimular el hambre de descubrimiento en los alumnos. Y en esto tienen un rol central (coadyuvantes y asociado a las tecnologías) diversos factores. Como toda moneda, la de las tecnologías pedagógicas también tiene dos caras. Pero en este caso no es recomendable revolearla para saber qué nos indica el azar, porque esas dos caras son los aspectos tecnológicos y los aspectos pedagógicos" (Gustavo, Abogado).

La mediación pedagógica, entonces, implica recobrar el sentido del proceso de enseñanza y de aprendizaje, tanto de los docentes como de los estudiantes.

Hablar, entonces de mediación es hablar de sentidos y significados, ubicándonos en una concepción de educación particular, si bien no nueva, pero que refuerza los aspectos críticos del proceso educativo: la participación del 
alumno como camino a la autonomía y autogestión, a su transformación individual y social, siendo protagonista en la comunicación y en el aprendizaje, con espacios para la creatividad, expresión y relación.

En torno a estas ideas una alumna recupera: "Las teorías más actuales de la educación conciben a ésta como un proceso que va mucho más allá de la simple transmisión de conocimientos, otorgando importancia fundamental a una dinámica y activa situación de diálogo entre docentes y alumnos y entre alumnos entre sí que posibilite el intercambio, construcción, debate y análisis del conocimiento... Hoy podemos, y entiendo tenemos el compromiso moral de hacerlo, proponer un modelo educativo basado en la colaboración, el diálogo y la construcción solidaria de experiencias y proyectos que nos enriquezcan mutuamente, utilizando para ellos las posibilidades que nos brindan los nuevos entornos comunicacionales" (Cecilia, Ing. Electrónica).

En este sentido, cobran relevancia las estrategias didácticas de intervención docente, las herramientas, los recursos, los medios de enseñanza que seleccionemos, desde las nociones de mediación simbólica y relacional, generando espacios educativos en una distancia transaccional óptima que resignifique el proceso de comunicación con la integración de las TIC.

Aquí nos parece importante rescatar la definición de (San Martín Alonso, 1995, p. 240) acerca de los materiales didácticos. Este autor los define como: "todos aquellos artefactos capaces de articular diferentes formas de representación simbólica (libros, videos, ilustraciones, etc.), instrumentos para la realización de diferentes tareas (observación, experimentos, cálculos, simulaciones, etc.) y los que sirven como referentes directos (objetos, modelos, etc.) que incorporados a las estrategias de enseñanza y aprendizaje coadyuvan a la reconstrucción del conocimiento aportando significaciones parciales de los conceptos y nociones que integran el currículum".

Por su parte, Escudero (citado en Área Moreira, 1998) nos dice que entenderemos como medios de enseñanza a "cualquier recurso tecnológico que articule en un determinado sistema de símbolos ciertos mensajes con propósitos educativos o informativos". El autor incluye en dicha categoría los medios gráficos (textos, manuales, el diario), los medios audiovisuales (video, cine y TV) y los medios informáticos.

Al respecto una alumna manifiesta: "Los medios de enseñanza que incluimos y utilizamos en nuestras clases y la forma en que orientamos a nuestros alumnos para aprovecharlos, influyen -por lo tanto- en los procesos cognitivos que realizarán... hay que diseñar las estrategias a poner en juego cuando se emplean recursos de este tipo, tanto para facilitar el acceso de los alumnos al medio, como para capacitar en el uso adecuado del mismo... Considero que, a pesar de todo lo que aún resta por trabajar, investigar y descubrir, la clave de por qué y cómo introducir tecnologías en la enseñanza reside en el docente. Si como tal espero que un alumno repita lo que le transmito, lo mismo da que 
se lo dicte para que tome notas o que le señale ciertas páginas web y de allí extraiga la información a repetir. Por lo tanto, no es, estrictamente, el uso de tecnología lo que permite aprendizajes de calidad; sin duda puede favorecerlos, pero su sola presencia no los garantiza. Sí puede hacerlo, en cambio, el entorno de aprendizaje que utilicemos: la conjunción de metas que nos proponemos -y proponemos a nuestros alumnos-, el diseño de actividades que hacemos, los recursos que consecuentemente utilizamos y los procesos de avaluación y evaluación que ponemos en juego. Porque las TIC constituyen herramientas y entornos. Pero es el docente quien debe diseñar -en vistas a los objetivos de aprendizaje a lograr- qué actividades se realizarán en esos entornos y con esas herramientas. Serán esas actividades la parte fundamental de las condiciones para favorecer el aprendizaje a que me refería. Tal vez sea ese el puente levadizo más difícil de construir. Por cierto que no será rápido de transitar" (María del Carmen, Prof. en Educación).

Estas definiciones nos hacen pensar reflexivamente que la incorporación de un recurso supone un momento especial en la toma de decisiones del proceso metodológico, es parte estructurante de la actividad de enseñanza, no se trata de un mero complemento y es necesario considerar en su elección las características propias de cada uno de ellos para ser recontextualizados en la propuesta de enseñanza.

\section{A modo de cierre}

Queremos, a modo de reflexión final, compartir algunas ideas que, nos parecen, deben seguir acompañando el diseño y desarrollo de prácticas educativas hoy:

- Son las intencionalidades y los contextos de uso en los que los docentes y los alumnos utilizan las herramientas, los recursos multimediales los que determinan el impacto de los mismos en las prácticas educativas y su capacidad de transformación de los procesos de enseñanza y de aprendizaje.

- En la consideración de que es en el entrecruzamiento del contenido; las actividades de enseñanza, los recursos, las formas de interacción que llevan a cabo docentes y alumnos, donde vamos a lograr poder realizar inclusiones potentes de las TIC en nuestras propuestas educativas.

- $\quad$ El potencial de las TIC para transformar, innovar o mejorar las propuestas educativas depende del planteamiento pedagógico/didáctico en el que se inserta su utilización. Son la visión y los pensamientos pedagógicos en torno a los procesos de enseñanza los que marcan las maneras de incluir los recursos multimediales en el diseño e implementación de las propuestas educativas. 
Las nuevas tecnologías han dado origen a nuevas formas culturales, nuevos modos de conocer y nuevas formas de aprender. Sin embargo, la relación pedagógica sigue y seguirá siendo insustituible.

\section{Notas Bibliográficas}

(1) Sobre esta temática sugerimos: De Zubiría Samper, J. (2006), Luzuriaga, L. (1992), Château, J. (1974), Stramiello, C. (2001), Gallino, M. (2005).

(2) Baquero, R., Camilloni, A. et al. (2001) "Debates constructivistas". Buenos Aires, Aique.

(3) Baquero, R., Camilloni, A. et al. (2001).

\section{Referencias Bibliográficas}

- Área Moreira, M. (2001) Educar en la Sociedad de la Información. Bilbao, Desclée.

- $\quad$ Área Moreira, M. (1998) "Los medios y materiales impresos en el curriculum", en Sancho Gil, J. (1998) Para una tecnología educativa (Coord.). España, Horsori.

- Baquero, R. y Naradowsky, M. (1990), "Normatividad y Normalidad en Pedagogía", en Revista Alternativas Año IV, № 6, Univ. Nac. del Centro, Tandil, pp. 35-46.

- Baquero, R., Camilloni, A. et al. (2001) Debates constructivistas. Buenos Aires, Aique.

- Bernstein, B. (1998) Pedagogía, control simbólico e identidad: teoría, investigación y crítica. España, Morata.

- Bowen, J. (1985) Historia de la Educación Occidental. Vol. 3. Barcelona, Herder.

- Burbules, N. y Callister, T. (2001) Educación: riesgos y promesas de las nuevas tecnologías de la información. España, Granica.

- Burbules, N. (1999) El diálogo en la enseñanza. Teoría y práctica. Buenos Aires, Amorrortu.

- Château, J. (1974) Los grandes pedagogos. México, Fondo de Cultura Económica.

- De Zubiría Samper, J. (2006). Los modelos pedagógicos. Hacía una pedagogía dialogante. Aula Abierta, Magisterio.

- Edelstein, G. (1997) "Un capítulo pendiente: el método en el debate didáctico contemporáneo", en Camilloni, A. et al. (1997) Corrientes didácticas contemporáneas. Buenos Aires, Paidós.

- Furlán, A. (1989) "Metodología de la enseñanza", en Aportaciones a la Educación Superior. México, UNAM.

- Gallino, M. (2005) Cuadernos de trabajo. Cátedra de Pedagogía. FCEFyN, UNC. 
- Gimeno Sacristán, J. (1998) El curriculum: una reflexión sobre la práctica. Madrid, Morata.

- Jackson, P. (2002) Práctica de enseñanza. Buenos Aires, Amorrortu.

- Lion, C. (2006) Imaginar con tecnologías. Relaciones entre tecnologías y conocimiento. Buenos Aires, Stella/La Crujía.

- Litwin, E. (2005) Tecnologías educativas en tiempos de Internet. Buenos Aires, Amorrortu.

- Luzuriaga, L. (1992) Ideas Pedagógicas del siglo XX (4ª . Ed.). Buenos Aires, Lozada/Mimeo.

- Sabulsky, G. (2004) "La construcción del conocimiento y la integración de los medios en el marco del proceso metodológico". Ponencia de concurso. FFyH. UNC.

- San Martín Alonso, A. (1995) La escuela de las tecnologías. Valencia, Educación Estudis 9.

- Sancho Gil, J. (2006) Tecnologías para transformar la educación. Madrid, Akal.

- Sarason, S. (2002) La enseñanza como arte de representación. Buenos Aires, Amorrortu.

- Stramiello, C. (2001) Tres momentos, tres educadores: la educación popular. Buenos Aires, Educa.

- Stramiello, C. (2001) La educación superior según J. H. Newman. Buenos Aires, Educa. 